\title{
Anaerobic biosynthesis of rhamnolipids by Pseudomonas aeruginosa: performance, mechanism and its application potential for enhanced oil recovery
}

\author{
Feng Zhao ${ }^{1 *} \mathbb{B}$, Qingzhi Wang ${ }^{1}$, Ying Zhang ${ }^{2}$ and Liying Lei ${ }^{2}$
}

\begin{abstract}
Background: Pseudomonas aeruginosa, the rhamnolipids-producer, is one of dominant bacteria in oil reservoirs. Although P. aeruginosa strains are facultative bacteria, the anaerobic biosynthesis mechanism of rhamnolipids is unclear. Considering the oxygen scarcity within oil reservoirs, revealing the anaerobic biosynthesis mechanism of rhamnolipids are significant for improving the in-situ production of rhamnolipids in oil reservoirs to enhance oil recovery.
\end{abstract}

Results: Pseudomonas aeruginosa SG anaerobically produced rhamnolipids using glycerol rather than glucose as carbon sources. Two possible hypotheses on anaerobic biosynthesis of rhamnolipids were proposed, the new anaerobic biosynthetic pathway (hypothesis 1) and the highly anaerobic expression of key genes (hypothesis 2). Knockout strain SG $\triangle r m \mid B$ failed to anaerobically produce rhamnolipids using glycerol. Comparative transcriptomics analysis results revealed that glucose inhibited the anaerobic expression of genes rmIBDAC, fabABG, rhIABRI, rh/C and lasl. Using glycerol as carbon source, the anaerobic expression of key genes in P. aeruginosa SG was significantly up-regulated. The anaerobic biosynthetic pathway of rhamnolipids in P. aeruginosa SG were confirmed, involving the gluconeogenesis from glycerol, the biosynthesis of dTDP-L-rhamnose and $\beta$-hydroxy fatty acids, and the rhamnosyl transfer process. The engineered strain P. aeruginosa PrhIAB constructed in previous work enhanced $9.67 \%$ of oil recovery higher than the wild-type strain P. aeruginosa SG enhancing $8.33 \%$ of oil recovery.

Conclusion: The highly anaerobic expression of key genes enables P. aeruginosa SG to anaerobically biosynthesize rhamnolipids. The genes, rmIBDAC, fabABG, rhIABRI, rh/C and lasl, are key genes for anaerobic biosynthesis of rhamnolipid by P. aeruginosa. Improving the anaerobic production of rhamnolipids better enhanced oil recovery in core flooding test. This study fills the gaps in the anaerobic biosynthesis mechanism of rhamnolipids. Results are significant for the metabolic engineering of $P$. aeruginosa to enhance anaerobic production of rhamnolipids.

Keywords: Pseudomonas aeruginosa, Rhamnolipids, Anaerobic biosynthesis, Glycerol, RmIBDAC, Microbial enhanced oil recovery

\footnotetext{
*Correspondence: zhaofeng2019@qfnu.edu.cn; zhao2008569@126.com ${ }^{1}$ School of Life Sciences, Qufu Normal University, Qufu 273165, Shandong Province, China

Full list of author information is available at the end of the article Correspondence to Feng Zhao.
}

\begin{abstract}
Background
At present, the demand for oil in economic development continues to increase, while the recoverable oil reserves in oil reservoirs are decreasing, and it is more difficult to find new oil and gas resources. It is of great strategic significance to enhance the oil recovery of existing oil fields
\end{abstract}

(c) The Author(s) 2021. This article is licensed under a Creative Commons Attribution 4.0 International License, which permits use, sharing, adaptation, distribution and reproduction in any medium or format, as long as you give appropriate credit to the original author(s) and the source, provide a link to the Creative Commons licence, and indicate if changes were made. The images or other third party material in this article are included in the article's Creative Commons licence, unless indicated otherwise in a credit line to the material. If material is not included in the article's Creative Commons licence and your intended use is not permitted by statutory regulation or exceeds the permitted use, you will need to obtain permission directly from the copyright holder. To view a copy of this licence, visit http://creativeco mmons.org/licenses/by/4.0/. The Creative Commons Public Domain Dedication waiver (http://creativecommons.org/publicdomain/ zero/1.0/) applies to the data made available in this article, unless otherwise stated in a credit line to the data. 
through scientific research and innovation. Microbial oil recovery technology (MEOR) is an economical, effective and environmentally friendly oil recovery technology [1]. It is feasible to enhance oil recovery using microorganisms to in-situ produce biosurfactants in oil reservoirs [2-4]. It is also proved that the stable microbial growth and efficient production of biosurfactants such as rhamnolipids in reservoirs are the keys to the successful implementation of MEOR [1, 3, 4]. Rhamnolipids can effectively reduce the oil-water interfacial tension, emulsify crude oil and change the wettability of reservoir rock, so it is one of the excellent oil displacement agents used to improve oil recovery $[1,4]$. The environments in oil reservoirs are anoxic or anaerobic [5]. The injection of air into oil reservoirs is difficult to ensure the effective oxygen supply of aerobic microorganisms [4]. However, studies on anaerobic synthesis of biosurfactants are still weak [6], which limits to enhance oil recovery by anaerobic production of biosurfactants in oil reservoirs.

Although rhamnolipids as a natural biosurfactants is extensively studied and applied in MEOR and other fields $[7,8]$, studies on anaerobic biosynthesis of rhamnolipids are also scarce [6]. Many rhamnolipid-producing strains have been repoted, such as Pseudomonas sp. and Burkholderia sp. [8]. Among the rhamnolipid-producing strains, Pseudomonas aeruginosa has the highest rhamnolipid yield [8]. Therefore, P. aeruginosa was mainly studied for producing rhamnolipids [8, 9]. P. aeruginosa are facultative bacterial strains that can grow and metabolize at both aerobic and anaerobic conditions $[6,10,11]$. But most of the studies on rhamnolipids production by $P$. aeruginosa were focused on aerobic biosynthesis.

Studies have discovered that some $P$. aeruginosa strains can anaerobically produce rhamnolipids when using glycerol as carbon source [12-15]. But the related anaerobic biosynthesis mechanisms of rhamnolipids are unclear. How to further enhance the anaerobic yield of rhamnolipids requires the modification of key genes or the regulation of the anaerobic biosynthetic pathways, which all depend on the anaerobic biosynthesis mechanisms of rhamnolipids.

This study aims to explore the underlying anaerobic biosynthetic mechanism of rhamnolipids when $P$. aeruginosa SG using glycerol as carbon source. Strain P. aeruginosa SG was used for rhamnolipids production [13, 14]. P. aeruginosa SG was cultured with different carbon sources (glucose and glycerol). Cell growth and rhamnolipids production were comparatively studied. Two possible hypotheses on anaerobic biosynthesis of rhamnolipid using glycerol were proposed. RNA sequencing (RNA-seq) was performed on the anaerobic culture of strain SG using glucose and glycerol as carbon source, respectively. The transcriptomic data were analyzed for the anaerobic biosynthesis pathways of rhamnolipids and related genes regulation. The method of gene knock-out was used to identify the key genes and the anaerobic biosynthetic pathway of rhamnolipids when $P$. aeruginosa SG using glycerol. Application potential by anaerobic production of rhamnolipids for MEOR was evaluated and discussed. Results will help to regulate $P$. aeruginosa to produce more rhamnolipids under anaerobic conditions. This study also provides scientific guidance for the development of MEOR technology through in situ production of rhamnolipids.

\section{Materials and methods}

\section{Bacterial strains and culture conditions}

Strain $P$. aeruginosa SG was used as a rhamnolipidsproducer in this study. $P$. aeruginosa SG can anaerobically produce rhamnolipids $[13,14]$. P. aeruginosa SG was cultured in LB (Luria-Bertani) medium to prepare seed culture at $35{ }^{\circ} \mathrm{C}$ and $180 \mathrm{rpm}$ for $12 \mathrm{~h}$. To avoid interference of rhamnolipids produced in seed culture, the seed culture was centrifuged at $5000 \mathrm{~g}$ for $2 \mathrm{~min}$. The bacterial cells were washed twice using sterile distilled water. The suspension liquid of bacterial cells with sterile distilled water was transferred into the fermentation medium. The fermentation medium except for carbon source contained $4 \mathrm{~g} / \mathrm{l}$ of $\mathrm{NaNO}_{3}, 3 \mathrm{~g} / \mathrm{l}$ of $\mathrm{KH}_{2} \mathrm{PO}_{4}, 4 \mathrm{~g} / \mathrm{l}$ of $\mathrm{K}_{2} \mathrm{HPO}_{4} \cdot 3 \mathrm{H}_{2} \mathrm{O}, 0.5 \mathrm{~g} / \mathrm{l}$ of $\mathrm{KCl}, 0.5 \mathrm{~g} / \mathrm{l}$ of NaCl, $0.2 \mathrm{~g} / \mathrm{l}$ of $\mathrm{CaCl}_{2} \cdot 2 \mathrm{H}_{2} \mathrm{O}, 1.0 \mathrm{~g} / \mathrm{l}$ of $\mathrm{MgSO}_{4} \cdot 7 \mathrm{H}_{2} \mathrm{O}$. Glucose and glycerol were used as carbon sources with the concentrations of $40 \mathrm{~g} / \mathrm{l}$. The aerobic culture was performed in $250 \mathrm{ml}$-triangular flasks containing $100 \mathrm{ml}$ fermentation medium, at $35^{\circ} \mathrm{C}$ and $180 \mathrm{rpm}$ for 6 days. The anaerobic culture was performed in $100 \mathrm{ml}$-serum bottles containing $80 \mathrm{ml}$ anaerobic fermentation medium. The anaerobic medium was prepared as described in previous studies $[13,16]$. The anaerobic culture was incubated at $35{ }^{\circ} \mathrm{C}$ and $50 \mathrm{rpm}$ for 8 days. Three parallels were set for each experiments. The inoculum amount was $3 \%(\mathrm{v} / \mathrm{v})$. The non-inoculated medium was used as the negative control. Escherichia coli $\mathrm{DH} 5 \alpha$, E. coli $\mathrm{S} 17-1$ and recombinant strains were cultured in LB medium at $35{ }^{\circ} \mathrm{C}$ and $180 \mathrm{rpm}$. During gene knock-out process, LB medium containing ampicillin of $100 \mathrm{mg} / \mathrm{l}$ and kanamycin of $50 \mathrm{mg} / \mathrm{l}$ were used for recombinant $E$. coli strains. LB medium containing kanamycin of $350 \mathrm{mg} / \mathrm{l}$ was used for recombinant $P$. aeruginosa strains.

\section{Analytical methods}

The anaerobic culture was sampled from serum bottles using sterile syringes. Cell growth of strain SG was represented by $\mathrm{OD}_{600}$ values in the anaerobic culture. Then samples were centrifuged at $10,000 \mathrm{~g}$ for $10 \mathrm{~min}$, respectively. The cell free supernatant was collected. The 
surfactants anaerobically produced by $P$. aeruginosa SG using glycerol were rhamnolipids which were confirmed by analytical methods of FTIR and HPLC-MS [14]. In this study, rhamnolipids concentration was also represented by the diameter of oil spreading circle formed by cell free supernatant. The diameter of oil spreading circle was measured as described in previous studies [17, 18]. Then residue supernatant surface tension was measured by surface tensiometer (BZY-1, Shanghai Hengping Instrument and Meter Factory, Shanghai, China).

\section{Gene rm/B knock-out}

In this study, using the suicidal plasmid pK18mobSacB of $P$. aeruginosa, gene $r m l B$ was knocked out to block the biosynthetic pathway of dTDP-L-rhamnose controlled by $r m l B D A C$ operon genes. The $r m l B D A C$ operon genes control the biosynthesis of dTDP-rhamnose [19]. The gene fragment rmlBD was obtained by PCR using the genomic DNA of $P$. aeruginosa SG as the template. The PCR primers were rmBD-f: $5^{\prime}$-CGGAAGCTTATGTGG ACCGCTCGAT-3' and rmBD-r: 5'-GCCGAATTCCTG TTGCAGCTTGCGGT-3'. The $r m l B D$ fragment was 1969 bp with restriction sites of Hind III and EcoR I at the $5^{\prime}$ end and $3^{\prime}$ end, respectively. The $r m l B D$ fragment was cloned into pMD19T (simple) vector to construct plasmid pMD19-rmlBD. The rmlBD fragment contains two Eco52 I restriction sites at $831 \mathrm{bp}$ and $1206 \mathrm{bp}$. After pMD19T-rmlBD successively digested with Eco52 I enzyme and T4 DNA ligase, plasmid pMD19- $\Delta$ rmlBD was constructed and purified by gel extraction kit (Takara, Japan). The $\Delta \mathrm{rmlBD}$ fragment was cloned into the Hind III and EcoR I sites of plasmid pK18mobSacB to construct recombinant plasmid pK18- $\Delta$ rmlBD. The recombinant plasmid pK18- $\Delta \mathrm{rmlBD}$ was transformed into strain $E$. coli S17-1. Using conjugation method [20], the plasmids pK18- $\Delta$ rmlBD were transferred into the strain $P$. aeruginosa SG. Mutants were cultured on LB medium containing Ampicillin and kanamycin for the first recombination. The LB medium containing 20\% sucrose and Ampicillin was used to pick out the knockout strain $P$. aeruginosa $\mathrm{SG} \Delta \mathrm{rmlB}$. The anaerobic cell growth and anaerobic rhamnolipid production of wildtype strain SG and the knockout strain $\mathrm{SG} \Delta \mathrm{rmlB}$ were comparatively determined.

\section{RNA extraction, library construction and RNA sequencing}

$P$. aeriginosa SG cells were sampled from its anaerobic culture with $\mathrm{OD}_{600}=0.6$. P. aeriginosa SG cultured with glucose and glycerol as carbon sources were marked as GluAn and GlyAn, respectively. Three replicates, GluAn1, GluAn-2, GluAn-3 and GlyAn-1, GlyAn-2, GlyAn-3, were sampled for GluAn and GlyAn. The bacterial cells were collected by centrifugation $\left(4{ }^{\circ} \mathrm{C}, 4000 g, 5 \mathrm{~min}\right)$.
The harvested bacterial cells were used for the total RNA extraction. The Takara RNA kit (Takara, Japan) was used for RNA extraction and purification according to the manufacturer provided protocol. Then RNA purity of GluAn samples and GlyAn samples was evaluated by Nanodrop2000 with $\mathrm{OD}_{260 / 280}$ values and $\mathrm{OD}_{260 / 230}$ values. RNA fragment length of GluAn samples and GlyAn samples was determined using Agilent 2100 Bioanalyzer. The tested mRNA was enriched by removing rRNA. After enrichment, mRNA is fragmented into short fragments. First strand of cDNA was synthesized by reverse transcription using random hexamers and mRNA as template, and then the buffer, dNTPs and DNA polymerase I were added to synthesize the two-strand cDNA. Then AMPure XP Beads were used to purify doublestranded cDNA. The purified double stranded cDNA was treated with terminal repair, addition of $A$ and joint. The cDNA libraries were constructed using the method of chain-specific library by the Allwegene BioTech in Beijing (China). RNA sequencing was performed using Illumina Hiseq 4000 with PE150 double terminal sequencing strategy by the Allwegene BioTech in Beijing (China).

\section{Transcriptome analysis}

The obtained raw reads were filtered to remove the reads with sequencing adapter and the low-quality Reads. The sequencing data were statistically analyzed for raw reads numbers, clean reads numbers, sequence error rate, Q20 and Q30 (proportion of clean data with Phred value greater than 20 and 30 ) and GC content (\%). The high quality data, clean reads, were used for transcriptome analysis [21]. The obtained clean reads were mapped to the reference genome of $P$. aeruginosa PAO1 using Bowtie2 alignment software. The regional distribution and Reads density distribution were evaluated. The gene annotation, gene structure analysis and the new transcript prediction were performed.

FPKM (Fragments per kilobase of exon model per million mapped reads) values were calculated using HTSeq software [22] for the gene expression level analysis of each sample. The number of genes and the expression level of a single gene at different expression levels were counted respectively, and the FPKM value of one was used as the threshold to judge whether the gene was expressed or not. Using DESeq method [23], gene differential expression analysis was performed according to the readCount data obtained from gene expression level analysis. The differentially expressed genes between GluAn samples and GlyAn samples were identified with $\log 2$ (FoldChange) value $>1$ and $q$-value $<0.05$. Hierarchical clustering analysis of differentially expressed genes was performed based on their expression levels, $\log 10(\mathrm{FPKM}+1)$. The GO (Gene Ontology, http://www. 
geneontology.org/) enrichment analysis was carried out using GOseq software [24], and the probability of GO term enriched by differential genes was calculated. The differentially expressed genes were enriched into molecular function, biological process, cellular component. KEGG (Kyoto Encyclopedia of Genes and Genomes, http://www.kegg.jp) enrichment analysis was performed for pathways enrichment of the differentially expressed genes [25].

\section{Pathways description and genes discovery}

The metabolic pathways related to fatty acid metabolism, glycometabolism and rhamnosyl transfer process were mined from the transcripts information that has be enriched into KEGG pathways. Referring to the known information of aerobic biosynthetic pathways of rhamnolipid in model strain $P$. aeruginosa PAO1, the anaerobic biosynthetic pathways of rhamnolipid were attempted to analyze, starting with the metabolism of glycerol. Two precursors of rhamnolipid biosynthesis, dTDPL-rhamnose and $\beta$-hydroxy fatty acids, were of particular consideration. In the transcriptome of $P$. aeruginosa SG using glycerol under anaerobic conditions, the anaerobic biosynthesis of dTDP-L-rhamnose and $\beta$-hydroxy fatty acids and the transfer process of rhamnosyl were the target biosynthetic pathways. When P. aeruginosa SG using glucose as carbon source under anaerobic conditions, the related biosynthetic pathways were also comparatively analyzed.

The transcriptome data of $P$. aeruginosa SG using glucose and glycerol as carbon source under anaerobic conditions were compared and analyzed. The related key genes that were significantly up-regulated or activated when using glycerol as carbon source were mined from the differentially expressed genes between GluAn samples and GlyAn samples. Referring to the known key genes of aerobic biosynthetic pathways of rhamnolipid in model strain $P$. aeruginosa PAO1, the candidate key genes involving in the anaerobic biosynthesis of rhamnolipid using glycerol were picked out from the significantly upregulated genes. The genes related to the biosynthesis of dTDP-L-rhamnose and $\beta$-hydroxy fatty acids, and the rhamnosyl transfer process were the target genes.

\section{Core flooding test}

Enhanced oil recovery by in-situ anaerobic production of rhamnolipids was evaluated by core flooding test. The experimental protocol of core flooding test was referred to the previous study [13]. The engineered strain $P$. aeruginosa PrhlAB with higher anaerobic yield of rhamnolipids was used in core flooding tests. The engineered strain $P$. aeruginosa PrhlAB was constructed by increasing the copy number of rhlAB genes in P. aeruginosa SG
[15]. The rock core has length of $293 \mathrm{~mm}$, diameter of $38 \mathrm{~mm}$, absolute permeability of $0.388 \mu \mathrm{m}^{2}$, pore volume of $69.9 \mathrm{ml}$, respectively. The Xinjiang oilfield production water and crude oil (density of $0.886 \mathrm{~g} / \mathrm{cm}^{3}$ and viscosity of $6.3 \mathrm{mPas}$ ) were used. After the first water flooding, $0.5 \mathrm{PV}$ of culture solution [strain PrhlAB and medium $(1: 20, \mathrm{v} / \mathrm{v})]$ was injected into rock core. The medium contained $40 \mathrm{~g} / \mathrm{l}$ glycerol, $4 \mathrm{~g} / \mathrm{l}$ of $\mathrm{NaNO}_{3}, 3 \mathrm{~g} / \mathrm{l}$ of $\mathrm{KH}_{2} \mathrm{PO}_{4}$, $4 \mathrm{~g} / \mathrm{l}$ of $\mathrm{K}_{2} \mathrm{HPO}_{4} \cdot 3 \mathrm{H}_{2} \mathrm{O}, 0.5 \mathrm{~g} / \mathrm{l}$ of $\mathrm{KCl}, 0.5 \mathrm{~g} / \mathrm{l}$ of NaCl , $0.2 \mathrm{~g} / \mathrm{l}$ of $\mathrm{CaCl}_{2} \cdot 2 \mathrm{H}_{2} \mathrm{O}$. Then the rock core was incubated at $39{ }^{\circ} \mathrm{C}$ for 8 days. During the core flooding process, the volumes of displaced oil $(\mathrm{ml})$ and displaced water $(\mathrm{ml})$ were recorded. Enhanced oil recovery efficiency (\%) was calculated.

\section{Results and discussion}

Rhamnolipids production and cell growth using glucose and glycerol

Under aerobic conditions, strain $P$. aeruginosa SG decreased the culture surface tension to $27.0 \mathrm{mN} / \mathrm{m}$ and $27.3 \mathrm{mN} / \mathrm{m}$ using both glucose and glycerol as carbon sources, respectively. The oil spreading circles diameters of the aerobic culture were $482 \mathrm{~mm}$ and $565 \mathrm{~mm}$ when P. aeruginosa SG using glucose and glycerol as carbon sources, respectively. The surface activity and oil spreading activity indicate that $P$. aeruginosa SG produced biosurfactants $[17,18]$. The produced biosurfactants by $P$. aeruginosa was rhamnolipids [14, 15]. Under aerobic conditions, $P$. aeruginosa SG produced rhamnolipids using both glucose and glycerol as carbon sources.

But rhamolipids production by strain SG under anaerobic conditions were quite different. Under anaerobic conditions, the cell growth $\left(\mathrm{OD}_{600}\right)$, surface tension and oil spreading circles diameters were shown in Fig. 1, From the biomass $\left(\mathrm{OD}_{600}\right)$ point of view, strain $P$. aeruginosa SG grew well under anaerobic conditions using both glucose and glycerol as carbon sources (Fig. 1a). However, the surface tension values of anaerobic culture were decreased from 64 to $30 \mathrm{mN} / \mathrm{m}$ using glycerol (Fig. 1b). While the surface tension values of anaerobic culture were decreased from 57 to $48 \mathrm{mN} / \mathrm{m}$ using glucose. Results indicated that strain $P$. aeruginosa SG produced biosurfactants when using glycerol as carbon source. As shown in Fig. 1c, the oil spreading circles diameters of anaerobic culture were $24 \mathrm{~mm}$ and $8 \mathrm{~mm}$ when strain $P$. aeruginosa SG using glucose or glycerol as carbon sources, respectively. The oil spreading activity also demonstrates that $P$. aeruginosa SG produced biosurfactants when using glycerol as carbon source $[17,18]$. The anaerobically produced biosurfactants by $P$. aeruginosa SG was identified as rhamnolipids [13]. Results confirmed that $P$. aeruginosa SG can anaerobically grow well using both glucose and glycerol, but $P$. aeruginosa SG anaerobically 

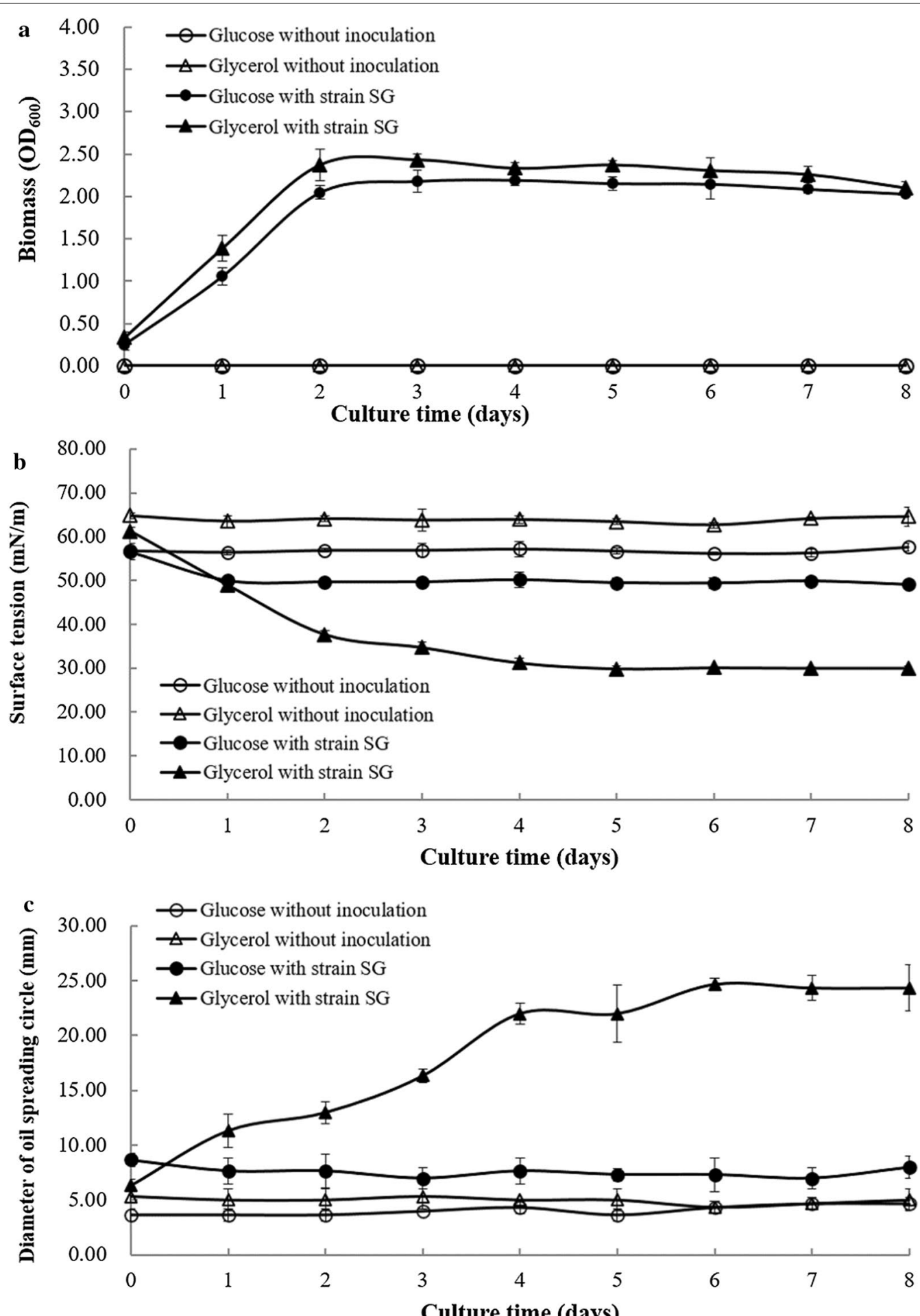

Fig. 1 Anaerobic growth and production of rhamnolipids by P. aeruginosa SG using glucose and glycerol as carbon sources a biomass $\left(\mathrm{OD}_{600}\right), \mathbf{b}$ surface tension and $\mathbf{c}$ oil spreading activity 
produces rhamnolipids when using glycerol as carbon sources.

\section{Research hypotheses for anaerobic biosynthesis of rhamnolipids using glycerol}

Under aerobic conditions, dTDP-rhamnose and $\beta$-hydroxy fatty acids are two required precursors for rhamnolipids biosynthesis [19]. The gluconeogenesis pathway, the Entner-Doudoroff pathway and de novo synthesis of fatty acids are all central metabolic pathways in $P$. aeruginosa. These metabolic pathways can also support the growth of $P$. aeruginosa under anaerobic conditions $[10,26]$. Therefore, under anaerobic conditions, glucose can be transformed into glucose-6-phosphate and then glucose-1-phosphate that was used for dTDPL-rhamnose biosynthesis. Through catabolic pathways, both glucose and glycerol can link with the de novo synthesis pathway of fatty acids under anaerobic conditions. And then $\beta$-hydroxy fatty acids can be synthesized by RhlA enzyme. Theoretically, the two precursors, dTDPL-rhamnose and $\beta$-hydroxy fatty acids, can be synthesize using both glucose and glycerol under anaerobic conditions. Therefore, rhamnolipids should be anaerobically synthesized by $P$. aeruginos $a$ using both glycerol and glucose as carbon sources. In fact, $P$. aeruginosa anaerobically synthesized rhamnolipids mere using glycerol rather than glucose as carbon source.

Here, two possible hypotheses on anaerobic biosynthesis of rhamnolipid by $P$. aeruginosa using glycerol were proposed. Hypothesis 1: in P. aeruginosa, the anaerobic biosynthetic pathway of rhamnolipid is different from the aerobic biosynthetic pathway of rhamnolipids. This hypothesis suggests that anaerobic conditions block the biosynthesis of dTDP-L-rhamnose from glucose1-phosphate, which results in $P$. aeruginosa being unable to anaerobically synthesize rhamnolipids from glucose. There may be other pathways to metabolize glycerol to synthesize dTDP-L-rhamnose without the effect of rmlBDAC operon genes, which leads to the anaerobic biosynthesis of rhamnolipids using glycerol. Hauser and Karnovsky (1957) cultured P. aeruginosa using ${ }^{14} \mathrm{C}$-labeled glycerol- $\alpha-{ }^{14} \mathrm{C}$ and glycerol- $\beta-{ }^{14} \mathrm{C}$ as carbon sources. They found that the $\mathrm{C}_{6}$ skeleton of rhamnose was composed of two ${ }^{14} \mathrm{C}$-labeled $\mathrm{C}_{3}$ units that were derived from two molecules glycerol $\left({ }^{14} \mathrm{C}\right.$-labeled $\mathrm{C}_{3}$ unit) without carbon chain rearrangement [27]. Hypothesis 2 was shown in Fig. 2. In P. aeruginosa, the rhamnolipids biosynthetic pathway is same under both aerobic and anaerobic conditions, but the anaerobic expression of key genes for rhamnolipids synthesis is inhibited using glucose as carbon source, and these key genes can be highly expressed under anaerobic conditions using glycerol as carbon source. Previous studies compared the gene expression differences of $P$. aeruginosa using glucose as carbon source under aerobic and anaerobic conditions, and the results showed that the expressions of $r h l R$, rhlI and rhlAB genes related to rhamnolipids synthesis were inhibited under anaerobic conditions using glucose [28-30].

From the two hypotheses point of view, the anaerobic synthesis of dTDP-rhamnose is one key metabolic pathway for rhamnolipids biosynthesis under anaerobic conditions. Knocking out the rmlBDAC operon can identify whether the $r m l B D A C$ genes involved in the anaerobic biosynthesis of rhamnolipid using glycerol. If the knockout strain $\mathrm{SG} \Delta \mathrm{rmlB}$ can anaerobically produce rhamnolipids using glycerol as carbon source, there is a new biosynthetic pathway of dTDP-L-rhamnose under anaerobic conditions (hypothesis 1). If not, the $\mathrm{rml}$ $B D A C$ genes control the anaerobic biosynthesis of dTDPrhamnose, and the expression of $r m l B D A C$ genes and other key genes are inhibited under anaerobic conditions (hypothesis 2). The transcriptomics results can provide the related information.

\section{Anaerobic production of rhamnolipids by strain SG and SG $\Delta$ rmIB using glycerol}

dTDP-Rhamnose and $\beta$-hydroxy fatty acids are two required precursors for rhamnolipids biosynthesis. The $r m l B D A C$ operon genes control the biosynthesis of dTDP-rhamnose, and $\beta$-hydroxy fatty acids are derived from the de novo synthesis of fatty acids [19]. The de novo synthesis of fatty acids is central metabolic pathway in $P$. aeruginosa. Therefore, anaerobic synthesis of dTDPrhamnose is one key metabolic pathway for rhamnolipid biosynthesis under anaerobic conditions.

The anaerobic cell growth and anaerobic rhamnolipids production of wild-type strain SG and the knockout strain $\mathrm{SG} \Delta \mathrm{rmlB}$ were comparatively determined. If the knockout strain $\mathrm{SG} \Delta \mathrm{rmlB}$ can anaerobically produce rhamnolipids using glycerol as carbon source, there is a new biosynthetic pathway of dTDP-L-rhamnose for rhamnolipids synthesis using glycerol under anaerobic conditions. If not, the $\operatorname{rmlBDAC}$ operon genes control the anaerobic biosynthesis of dTDP-rhamnose then for rhamnolipids synthesis using glycerol under anaerobic conditions. As shown in Fig. 3, the knockout strain $\mathrm{SG} \Delta \mathrm{rmlB}$ can anaerobically grow using both glucose and glycerol as carbon sources with $\mathrm{OD}_{600}=2.18$ and 2.33 . The wild-type strain SG can anaerobically produce rhamnolipids using glycerol as carbon source, reducing surface tension to $29.8 \mathrm{mN} / \mathrm{m}$ and forming oil spreading circle with diameter of $22.0 \mathrm{~mm}$. Although glycerol used as carbon source, the knockout strain SG $\Delta \mathrm{rmlB}$ can not anaerobically produce rhamnolipids, reducing surface tension to $48.9 \mathrm{mN} / \mathrm{m}$ and forming oil spreading circle 
$\mathbf{a}$

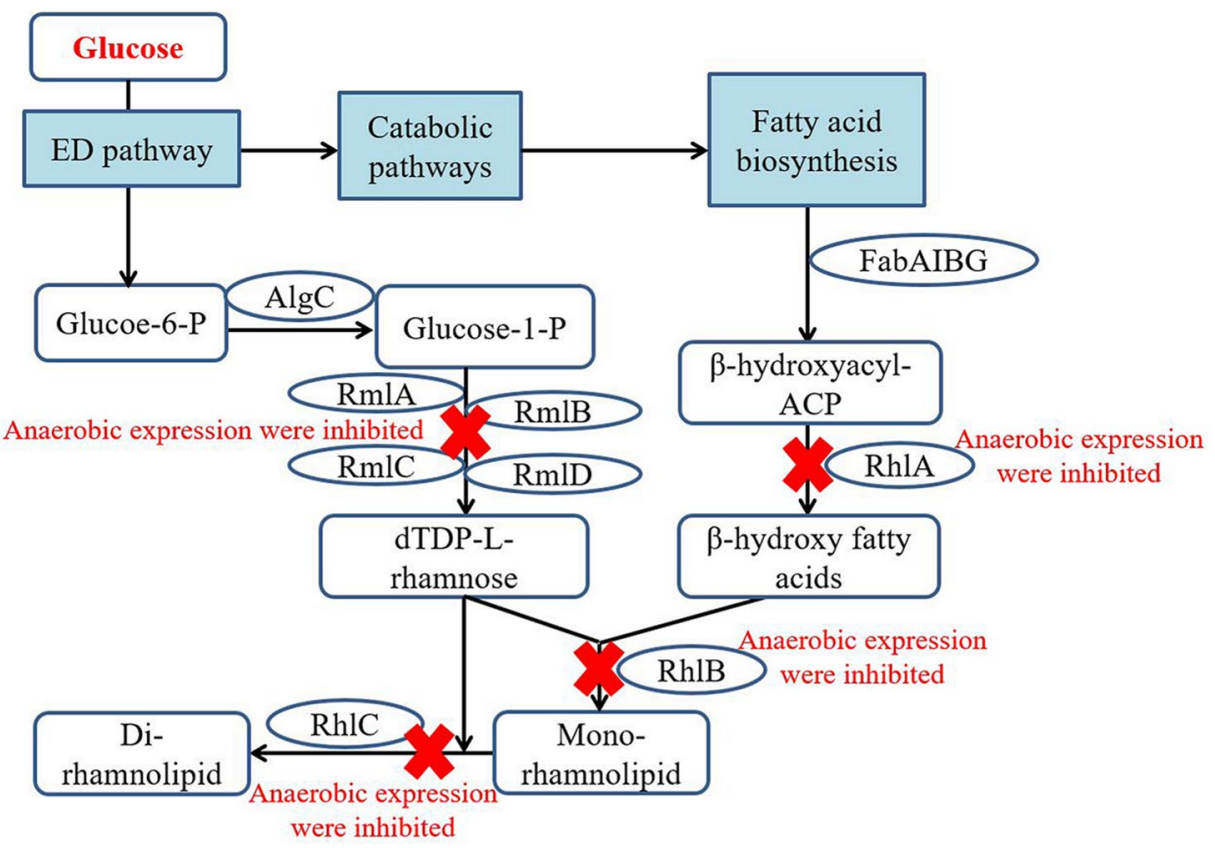

\section{b Glycerol}

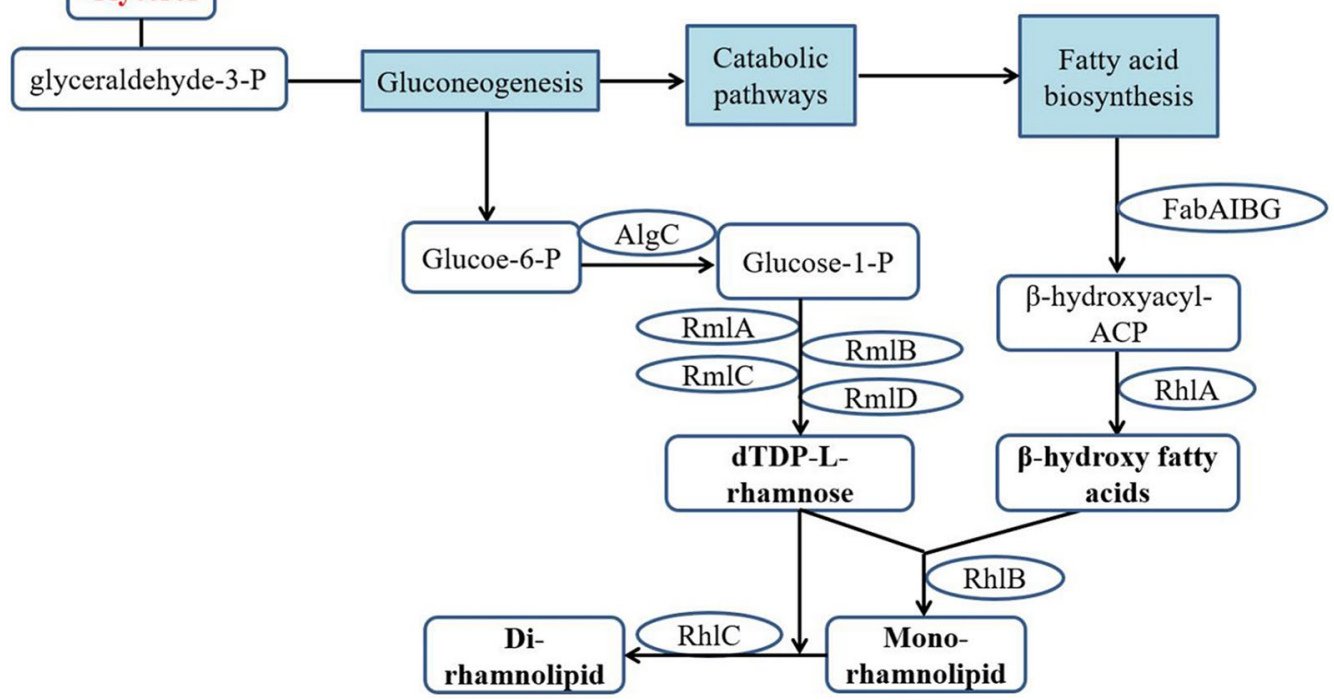

Fig. 2 The anaerobic biosynthetic pathways of rhamnolipid in P. aeruginosa SG a using glucose as carbon source; b using glycerol as carbon source

with diameter of $9.3 \mathrm{~mm}$. Results indicated that the $\mathrm{rml}$ $B D A C$ operon genes involved in the anaerobic biosynthesis of rhamnolipids using glycerol.

\section{Basic transcriptome results of strain SG using glycerol or glucose under anaerobic conditions}

To understand why glycerol can be used for anaerobically synthesizing rhamnolipids by strain SG while glucose cannot, RNA sequencing (RNA-seq) was performed on strain SG using glucose and glycerol as carbon source under anaerobic conditions, respectively. The RNA-seq data were shown in Table 1. A total of 90270732 Raw reads were obtained. After quality control, 84703416 Clean Reads were obtained. Totally, 5875 genes were mapped to the reference genome of $P$. aeruginosa PAO1 (NC_002516). The RNA sequencing data have been deposited in NCBI's Sequence Read Archive (SRA) with Accession Number PRJNA670563 (https:// 


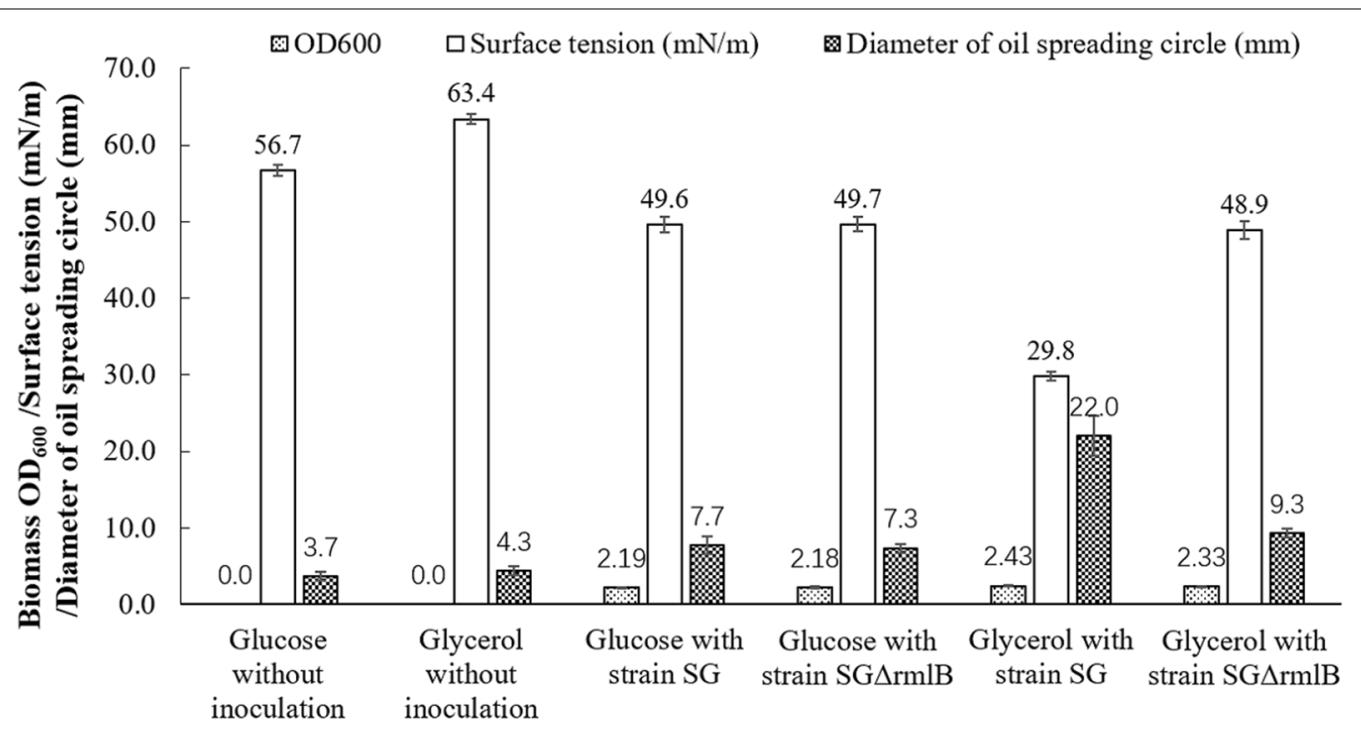

Stains and carbon sources

Fig. 3 Anaerobic growth and production of rhamnolipids by the wild-type strain P. aeruginosa SG and the knockout strain P. aeruginosa SG $\Delta r \mathrm{mlB}$ using glucose and glycerol as carbon sources

Table 1 RNA-seq data of strain SG using glycerol and glucose as carbon sources under anaerobic conditions

\begin{tabular}{lllllll}
\hline Sample name & Raw reads & Clean reads & $\begin{array}{l}\text { Clean bases } \\
(\mathbf{G b})\end{array}$ & GC content (\%) & Total mapped reads & $\begin{array}{l}\text { Mapped } \\
\text { percentage } \\
(\%)\end{array}$ \\
\hline GluAn-1 & 13878806 & 12993448 & 1.94 & 63.60 & 12542479 & 96.53 \\
GluAn-2 & 16251408 & 15319168 & 2.30 & 63.86 & 14794181 & 96.57 \\
GluAn-3 & 14265600 & 13323478 & 2.00 & 63.58 & 12748319 & 95.68 \\
GlyAn-1 & 16223066 & 15051700 & 2.26 & 64.14 & 14573577 & 96.82 \\
GlyAn-2 & 14887764 & 13860002 & 2.08 & 63.94 & 13433828 & 96.93 \\
GlyAn-3 & 14764088 & 14155620 & 2.12 & 63.94 & 13733081 & 97.02 \\
\hline
\end{tabular}

dataview.ncbi.nlm.nih.gov/object/PRJNA670563? reviewer $=q 57 \mathrm{rp} 98 \mathrm{cvqi} 8 \mathrm{nvid6dv8urgint}) . \quad$ FPKM values of the mapped genes were calculated for analysis of the gene expression level. The FPKM value of one was used as the threshold to judge whether the gene was expressed or not. The correlation of gene expression levels between samples is an important indicator to test the reliability of experiments and the reasonableness of sample selection [31]. The closer the Pearson correlation coefficient squared $\left(R^{2}\right)$ is to 1 , the higher the similarity of expression patterns between samples is and the less different genes among samples. In this study, Pearson correlation coefficient squared $\left(R^{2}\right)$ between samples in GluAn and samples in GlyAn were all greater than 0.92 , which indicated the ideal sampling and experimental conditions.
According to the readCount data obtained from gene expression level analysis, a total of 972 significantly differentially expressed genes with log2(FoldChange) value $>1$ and $\mathrm{q}$-value $<0.05$ were identified between strain $P$. aeruginosa SG using glycerol and glucose as carbon sources under anaerobic conditions. There were 549 up regulated genes and 423 down regulated genes in GlyAn samples vs GluAn samples. As shown in Fig. $4 \mathrm{a}$, the up regulated genes were significantly enriched into $37 \mathrm{GO}$ terms belonging to two major functional categrories, molecular function and biological process. KEGG pathways enrichment analysis showed that the up regulated genes were enriched into 20 pathways (Fig. 4b). Some of the enriched metabolic pathways are closely related to the biosynthesis of rhamnolipids, such as fatty acid biosynthesis and metabolism, quorum sensing, gluconeogenesis. 
$\mathbf{a}$

cellular carbohydrate metaboli... $O$ antigen biosynthetic process $\mathrm{O}$ antigen metabolic process cellular polysaccharide biosyn... cellular carbohydrate biosynth... cellular polysaccharide metabo... lipid biosynthetic process polysaccharide biosynthetic pr... monocarboxylic acid metabolic ... lipopolysaccharide metabolic $p . .$. lipopolysaccharide biosyntheti... carbohydrate metabolic process carbohydrate biosynthetic proc... cellular respiration extracellular polysaccharide b... extracellular polysaccharide $m$... energy derivation by oxidation... nucleotide-sugar metabolic pro... glycine betaine catabolic proc... polysaccharide metabolic proce... aromatic compound biosynthetic... nucleotide-sugar biosynthetic ... single-species biofilm formati... generation of precursor metabo... lipid metabolic process cellular modified amino acid c... phenazine biosynthetic process cellular aromatic compound met... fatty acid synthase activity

The Most Enriched GO Terms GlyAnvsGluAn

b

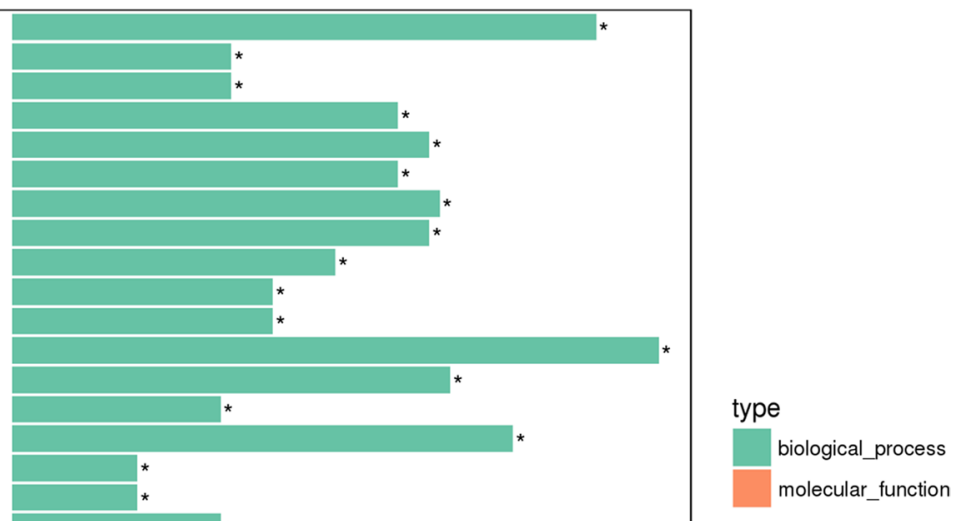

Statistics of Pathway Enrichment GlyAnvsGluAn

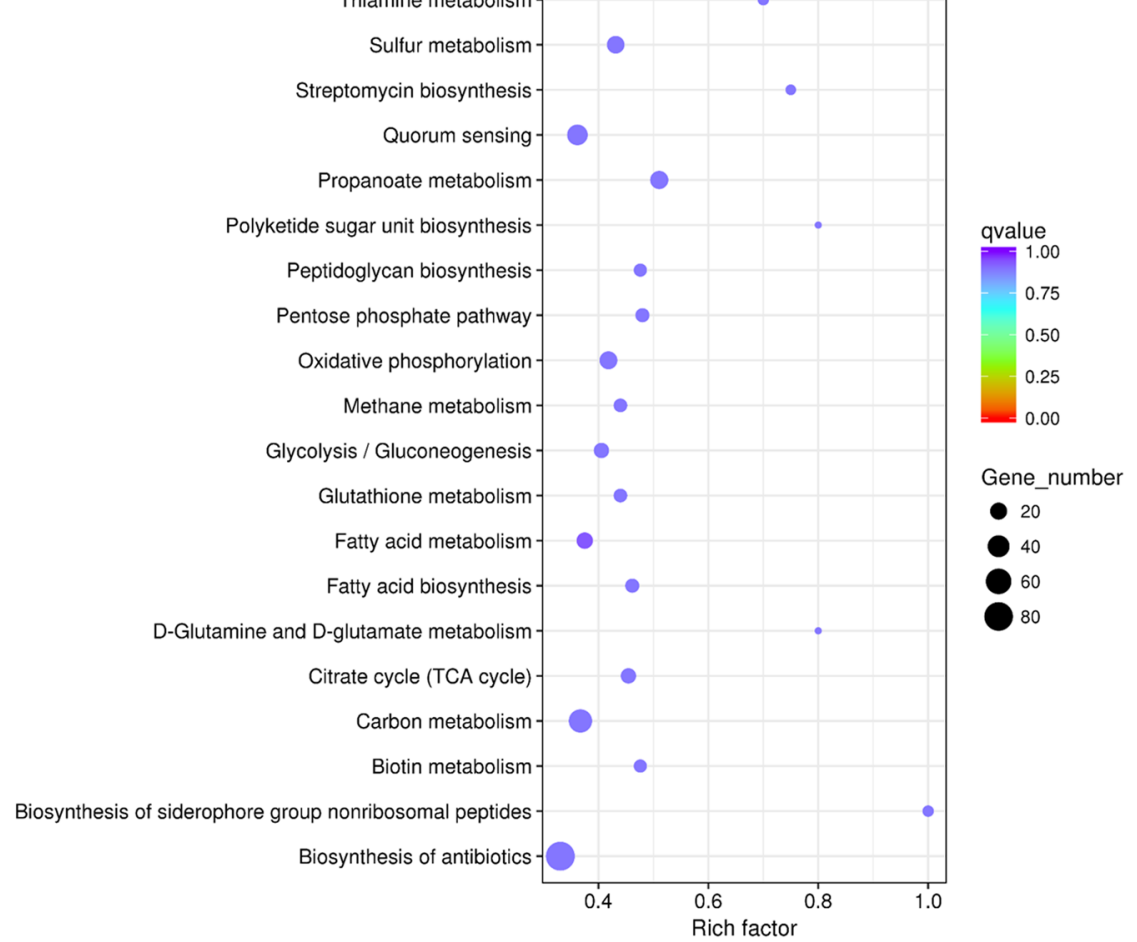

Fig. $4 \mathrm{GO}$ annotation and KEGG pathways enrichment of the differentially expressed genes between using GlyAn and GluAn a the most enriched GO Terms, the asterisk indicated that the GO item was significantly enriched $(P<0.05)$; and $\mathbf{b}$ the statistics of KEGG pathways enrichment 
Differentially expressed genes involved in anaerobic biosynthesis of rhamnolipids

As listed in Table 2, the genes related to the biosynthesis of dTDP-L-rhamnose and $\beta$-hydroxy fatty acids, and the rhamnosyl transfer process pathways were picked out from the differentially expressed genes between GlyAn samples and GluAn samples. The precursor, dTDP-L-rhamnose, provides the glycosyl part for the biosynthesis of rhamnolipids. It can be initiated by the gluconeogenesis pathway or the Entner-Doudoroff pathway, and the $\operatorname{alg} C$ gene and $r m l B D A C$ operon genes control the dTDP-L-rhamnose biosynthesis $[32,33]$. The another precursor, $\beta$-hydroxy fatty acids, provides the lipid chain part for the biosynthesis of rhamnolipids. It can be initiated by the de novo synthesis of fatty acids. Fatty acid synthase system II, Ketolipoyl reductase (FabG) and peptide-transferase (RhlA) catalyze the biosynthesis of $\beta$-hydroxy fatty acids [32, 33]. The two precursors, dTDPL-rhamnose and $\beta$-hydroxy fatty acids, were assembled to mono-rhamnolipids and di-rhamnolipids catalyzed by rhamnotransferase I (RhlB) and rhamnotransferase II (RhlC), respectively [34]. Rhamnotransferase I (RhlB) and rhamnotransferase II (RhlC) were coded by genes $r h l B$ and $r h l C$, respectively. The genes lasRI and $r h l R I$ in quorum-sensing pathways are responsible for activating the expression of genes, $\operatorname{rhlAB}$ and $\operatorname{rhlC}[34,35]$. Under anaerobic conditions, the related structural genes and regulatory genes, $\operatorname{rmlBDAC}, \operatorname{rhl} A B, \operatorname{rhlC}, \mathrm{fabA}$, $f a b G$ and rhlRI, lasI, were significantly up-regulated or activated when using glycerol as carbon source. Glycerol metabolism up-regulated the expression of genes in Quorum-sensing system, which promote the expression of genes $r m l B D A C, f a b A, f a b G, r h l A B$ and $r h l C$. The anaerobic high expression of key genes is significant for $P$. aeruginosa producing rhamnolipids under anaerobic conditions.

\section{Pathways and key genes for anaerobic synthesis of rhamnolipids using glycerol}

In this study, the knockout strain SG $\Delta$ rmlB could not anaerobically synthesize rhamnolipids even using glycerol as carbon source. Results showed that the pathways to metabolize glycerol to synthesize dTDP-L-rhamnose was also controlled by $\operatorname{rmlBDAC}$ operon genes under anaerobic conditions. The gene expression results showed that the related genes for rhamnolipids biosynthesis were highly expressed under anaerobic conditions using glycerol. The genes, $\operatorname{rmlBDAC}, \operatorname{rhl} A B, \operatorname{rhl} C, \mathrm{fab} A$, $f a b G$ and rhlRI, lasI, were significantly up-regulated expression using glycerol as carbon source, compared to using glucose under anaerobic conditions.

Based on the above results, Hypothesis 2 is more convincing (Fig. 2). The anaerobic biosynthesis mechanism of rhamnolipids in $P$. aeruginosa was revealed at the gene level using glycerol as carbon source. The anaerobic biosynthetic pathway of rhamnolipids in $P$. aeruginosa SG were confirmed, involving the gluconeogenesis from glycerol, the biosynthesis of dTDP-L-rhamnose and

Table 2 The differentially expressed genes related to the biosynthesis of dTDP-L-rhamnose and $\beta$-hydroxy fatty acids, and the rhamnosyl transfer process

\begin{tabular}{|c|c|c|c|c|c|c|}
\hline Gene id & Gene name & Readcount-GlyAn & Readcount-GluAn & $\log 2$ (foldchange) & p-value & Gene description \\
\hline PA5322 & $\operatorname{alg} C$ & 3951.9 & 3245.6 & 0.28 & 0.43 & phosphomannomutase AlgC \\
\hline PA5161 & $r m / B$ & 4139.6 & 1464.7 & 1.49 & $9.58 \mathrm{E}-09$ & dTDP-D-glucose 4,6-dehydratase \\
\hline PA5162 & $r m / D$ & 1650.9 & 538.10 & 1.62 & $1.02 \mathrm{E}-09$ & dTDP-4-dehydrorhamnose reductase \\
\hline PA5163 & $r m / A$ & 2270.2 & 789.6 & 1.52 & 8.69E-09 & glucose-1-phosphate thymidylyltransferase \\
\hline PA5164 & $\mathrm{rm} / \mathrm{C}$ & 2170.5 & 649.9 & 1.74 & $3.76 \mathrm{E}-11$ & dTDP-4-dehydrorhamnose 3,5-epimerase \\
\hline PA1609 & $f a b B$ & 2195.5 & 1304.6 & 0.75 & $1.65 \mathrm{E}-03$ & beta-ketoacyl-ACP synthase I \\
\hline PA1610 & $f a b A$ & 945.2 & 472.6 & 1.00 & $3.96 \mathrm{E}-05$ & beta-hydroxydecanoyl-ACP dehydrase \\
\hline PA2965 & $f a b F 1$ & 1117.3 & 966.8 & 0.21 & 0.40 & beta-ketoacyl-acyl carrier protein synthase II \\
\hline PA1373 & fabF2 & 109.6 & 395.1 & -1.85 & $2.57 \mathrm{E}-12$ & 3-oxoacyl-acyl carrier protein synthase II \\
\hline PA2968 & $f a b D$ & 702.6 & 439.7 & 0.68 & $6.68 \mathrm{E}-03$ & malonyl-CoA-[acyl-carrier-protein] transacylase \\
\hline PA2967 & $f a b G$ & 2143.7 & 926.5 & 1.21 & $5.82 \mathrm{E}-07$ & 3-oxoacyl-[acyl-carrier-protein] reductase \\
\hline PA3333 & $\mathrm{fabH} 2$ & 677.1 & 10.0 & 6.08 & $2.65 E-70$ & 3-oxoacyl-[acyl-carrier-protein] synthase III \\
\hline PA3476 & $r h l l$ & 5429.4 & 92.4 & 5.88 & $1.56 \mathrm{E}-87$ & autoinducer synthesis protein Rhll \\
\hline PA3477 & $r h / R$ & 13664.1 & 732.3 & 4.22 & $1.93 \mathrm{E}-56$ & transcriptional regulator RhIR \\
\hline PA3478 & $r h \mid B$ & 26462.6 & 243.7 & 6.76 & $1.28 \mathrm{E}-91$ & rhamnosyltransferase chain B \\
\hline PA3479 & rhlA & 42259.3 & 370.3 & 6.83 & $1.27 \mathrm{E}-69$ & rhamnosyltransferase chain A \\
\hline PA1130 & $r h / C$ & 1418.9 & 16.1 & 6.46 & $2.61 \mathrm{E}-86$ & rhamnosyltransferase 2 \\
\hline PA1432 & lasl & 1260.4 & 196.1 & 2.68 & $1.95 \mathrm{E}-23$ & autoinducer synthesis protein Lasl \\
\hline
\end{tabular}


$\beta$-hydroxy fatty acids, and the rhamnosyl transfer process, which is consistent with the aerobic biosynthetic pathways in model strain $P$. aeruginosa PAO1. The key genes involved in anaerobic biosynthesis of rhamnolipids are $r m l B D A C$, rhlABRI, rhlC, fabA, fabG and lasI. Glycerol metabolism up-regulated the expression of genes in Quorum-sensing system (rhlRI and lasI), which promote the expression of genes $r m l B D A C$, $r h l A B$ and $r h l C$, thus enable $P$. aeruginosa to anaerobically produce rhamnolipids using glycerol.

\section{Enhanced oil recovery by anaerobic production of rhamnolipids}

After the first water flooding, $45.21 \%$ of the oil was recovered from the core model. At the end of the second water flooding, 54.88\% of oil was recovered. In-situ anaerobic production of rhamnolipids by engineered strain $P$. aeruginosa PrhlAB enhanced $9.67 \%$ of oil recovery. In our previous study [13], in-situ anaerobic production of rhamnolipids by wild strain $P$. aeruginosa SG enhanced $8.33 \%$ of oil recovery. Increasing the copy number of rhlAB genes in $P$. aeruginosa $\mathrm{SG}$, the engineered strain $P$. aeruginosa PrhlAB can produce more rhamnolipids under anaerobic conditions [15]. Improving the anaerobic production of rhamnolipids better enhanced oil recovery in core flooding test. Future research will be focused on further enhancing the anaerobic production yield based on the anaerobic biosynthetic mechanism of rhamnolipids.

\section{Other prospect of the anaerobic production of rhamnolipids}

$P$. aeruginosa strains are facultative bacteria [10], but the vast majority of studies on the biosynthesis of rhamnolipids in $P$. aeruginosa were focused on aerobic biosynthesis $[19,34,35]$. Anaerobic biosynthesis of rhamnolipids is promising to the in-situ applications in anoxic environments, such as deep soil, oil reservoirs, sediments [4, 6 , 13]. Rhamnolipids products have also been partially commercialized in some fields. Nevertheless, the necessary aeration and defoaming processes in the aerobic fermentation of rhamnolipids lead to high production costs, which affects the large-scale industrial application of rhamnolipids $[12,36]$. The foam problem in production of rhamnolipids can be avoided by anaerobic fermentation without aeration. The low anaerobic production yield of rhamnolipids was the bottleneck for in-situ enhanced oil recovery, and the foamless fermentation. Enhancing the anaerobic production yield of rhamnolipids is of great importance. In the future, the revealed biosynthetic pathways and key genes involved in anaerobic biosynthesis of rhamnolipids would be regulated and modified to enhance $P$. aeruginosa to anaerobically produce more rhamnolipids. Besides, $\beta$-hydroxy fatty acids are precursor substrates of rhamnolipids synthesis as well. Fatty acid synthesis requires a lot of reducing power (NADPH or $\mathrm{NADH}_{2}$ ). Compared with anaerobic conditions, aerobic conditions are conducive to cell synthesis of reducing power, which promotes fatty acid synthesis and ultimately enhances rhamnolipids synthesis. Increasing the reducing power level in P. aeruginosa cells will also enhance the production of rhamnolipids under anaerobic conditions. Results of this study would be significant for the metabolic engineering of $P$. aeruginosa to enhance anaerobic production of rhamnolipids.

\section{Conclusion}

P. aeruginosa can anaerobically produce rhamnolipid using glycerol rather than glucose. Two possible hypotheses on anaerobic biosynthesis of rhamnolipid using glycerol were proposed. The $r m l B D A C$ operon genes involved in the anaerobic biosynthesis of rhamnolipid using glycerol. The expression of key genes was inhibited under anaerobic conditions when $P$. aeruginosa using glucose. The highly anaerobic expression of key genes enables $P$. aeruginosa to anaerobically biosynthesize rhamnolipids using glycerol. The anaerobic biosynthetic pathway of rhamnolipid using glycerol was confirmed. Improving the anaerobic production of rhamnolipids better enhanced oil recovery. This study fills the gaps in the anaerobic biosynthesis mechanism of rhamnolipids.

\section{Acknowledgements \\ This work was financially mainly supported by the National Natural Science Foundation of China (31700117). We thank Dr. Qingfeng Cui for his kind help in core flooding tests.}

\section{Authors' contributions}

FZ conceived and designed the study, carried out the experiments and drafted the manuscript. QZW assisted in bacterial cultivation and data analysis YZ assisted in analyzing the RNA-seq data. LYL participated in gene knockout experiments. All authors read and approved the final manuscript.

\section{Funding}

This work was financially supported by the National Natural Science Foundation of China (31700117) and the Introduced Talent Research Start-up Fund of Qufu Normal University, and the Young Talents Invitation Program of Shandong Provincial Colleges and Universities.

\section{Availability of data and materials}

The datasets supporting the conclusions of this article are included within the article.

\section{Declarations}

Ethics approval and consent to participate

Not applicable.

Consent for publication

Not applicable.

Competing interests

The authors declare that they have no competing interests. 


\section{Author details}

${ }^{1}$ School of Life Sciences, Qufu Normal University, Qufu 273165, Shandong Province, China. ${ }^{2}$ Institute of Applied Ecology, Chinese Academy of Sciences, Shenyang 110016, Liaoning Province, China.

Received: 4 March 2021 Accepted: 8 May 2021

Published online: 20 May 2021

\section{References}

1. Brown LR. Microbial enhanced oil recovery (MEOR). Curr opin Microbiol. 2010;13(3):316-20.

2. Youssef NH, Simpson DR, Duncan KE, et al. In situ biosurfactant production by Bacillus strains injected into a limestone petroleum reservoir. Appl Environ Microbiol. 2007;73(4):1239-47.

3. Youssef NH, Simpson DR, Mclnerney MJ, et al. In-situ lipopeptide biosurfactant production by Bacillus strains correlates with improved oil recovery in two oil wells approaching their economic limit of production. Int Biodeterior Biodegrad. 2013;81:127-32.

4. Zhao F, Li P, Guo C, Shi R, Zhang Y. Bioaugmentation of oil reservoir indigenous Pseudomonas aeruginosa to enhance oil recovery through in-situ biosurfactant production without air injection. Bioresour Technol. 2018;251:295-302.

5. Bian XY, Mbadinga SM, Liu YF, Yang SZ, Liu JF, Ye RQ, Gu JD, Mu BZ. Insights into the anaerobic biodegradation pathway of n-alkanes in oil reservoirs by detection of signature metabolites. Sci Rep. 2015;5(1):1-12

6. Domingues PM, Almeida A, Leal LS, et al. Bacterial production of biosurfactants under microaerobic and anaerobic conditions. Rev Environ Sci Bio. 2017;16(2):239-72.

7. Zou H, Du W, Ji M, Zhu R. Enhanced electrokinetic remediation of pyrenecontaminated soil through $\mathrm{pH}$ control and rhamnolipids addition. Environ Eng Sci. 2016;33(7):507-13.

8. Chong H, Li Q. Microbial production of rhamnolipids: opportunities, challenges and strategies. Microb Cell Fact. 2017;16(1):137.

9. Müller MM, Kügler JH, Henkel M, Gerlitzki M, Hörmann B, Pöhnlein M, Syldatk C, Hausmann R. Rhamnolipids-next generation surfactants? J Biotechnol. 2012;162:366-80.

10. Arai $H$. Regulation and function of versatile aerobic and anoxic respiratory metabolism in Pseudomonas aeruginosa. Front Microbiol. 2011;2:1-13.

11. Schobert M, Jahn D. Anaerobic physiology of Pseudomonas aeruginosa in the cystic fibrosis lung. Int J Med Microbiol. 2010;300:549-56.

12. Chayabutra C, Wu J, Ju LK. Rhamnolipids production by Pseudomonas aeruginosa under denitrification: effects of limiting nutrients and carbon substrates. Biotechnol Bioeng. 2001;72:25-33.

13. Zhao F, Zhang J, Shi R, Han S, Ma F, Zhang Y. Production of biosurfactant by a Pseudomonas aeruginosa isolate and its applicability to in situ microbial enhanced oil recovery under anoxic conditions. RSC Adv. 2015;5(45):36044-50.

14. Zhao F, Shi R, Ma F, Han S, Zhang Y. Oxygen effects on rhamnolipids production by Pseudomonas aeruginosa. Microb Cell Fact. 2018;17(1):39.

15. Zhao F, Cui Q, Han S, Dong H, Zhang J, Ma F, Zhang Y. Enhanced rhamnolipid production of Pseudomonas aeruginosa SG by increasing copy number of $r$ IAB genes with modified promoter. RSC Adv. 2015;5(86):70546-52.

16. Javaheri M, Jenneman GE, McInerney MJ, Knapp RM. Anaerobic production of a biosurfactants by Bacillus licheniformis JF-2. Appl Environ Microbiol. 1985;50(3):698-700.

17. Youssef NH, Duncan KE, Nagle DP, Savage KN, Knapp RM, Mclnerney MJ. Comparison of methods to detect biosurfactant production by diverse microorganisms. J Microbiol Methods. 2004;56:339-47.

18. Zhao F, Liang X, Ban Y, Han S, Zhang J, Zhang Y, Ma F. Comparison of methods to quantify rhamnolipids and optimization of oil spreading method. Tenside Surfact Det. 2016;53(3):243-8.
19. Soberón-Chávez G, Lepine F, Déziel E. Production of rhamnolipids by Pseudomonas aeruginosa. Appl Microbiol Biotechnol. 2005;68:718-25.

20. Schäfer A, Tauch A, Jäger W, Kalinowski J, Thierbach G, Pühler A. Small mobilizable multi-purpose cloning vectors derived from the Escherichia coli plasmids pK18 and pK19: selection of defined deletions in the chromosome of Corynebacterium glutamicum. Gene. 1994;145:69-73.

21. Nagalakshmi U, Waern K, Snyder M. RNA-Seq: a method for comprehensive transcriptome analysis. Curr Prot Mole Biol. 2010;89(1):4-11.

22. Anders S, Pyl PT, Huber W. HTSeq-a python framework to work with highthroughput sequencing data. Bioinformatics. 2014;31(2):166-9.

23. Anders $S$, Huber W. Differential expression analysis for sequence count data. Genome Biol. 2010;11(10):R106.

24. Young MD, Wakefield MJ, Smyth GK, et al. Gene ontology analysis for RNA-seq: accounting for selection bias. Genome Biol. 2010;11(2):R14.

25. Kanehisa M, Araki M, Goto S, et al. KEGG for linking genomes to life and the environment. Nucleic Acids Res. 2008;36(suppl 1):480-4.

26. Hunt JC, Phibbs PV. Regulation of alternate peripheral pathways of glucose catabolism during aerobic and anaerobic growth of Pseudomonas aeruginosa. J Bacteriol. 1983;154(2):793-802.

27. Hauser G, Karnovsky ML. Rhamnose and rhamnolipide biosynthesis by Pseudomonas aeruginosa. J Biol Chem. 1957;224(1):91-105.

28. Filiatrault MJ, Wagner VE, Bushnell D, Haidaris CG, Iglewski BH, Passador L. Effect of anaerobiosis and nitrate on gene expression in Pseudomonas aeruginosa. Infect Immun. 2005;73(6):3764-72.

29. Wagner VE, Bushnell D, Passador $L$, et al. Microarray analysis of Pseudomonas aeruginosa quorum-sensing regulons: effects of growth phase and environment. J Bacteriol. 2003;185(7):2080-95.

30. Alvarez-Ortega C, Harwood CS. Responses of Pseudomonas aeruginosa to low oxygen indicate that growth in the cystic fibrosis lung is by aerobic respiration. Mol Microbiol. 2007;65(1):153-65.

31. Hansen KD, Wu Z, Irizarry RA, Leek JT. Sequencing technology does not eliminate biological variability. Nat Biotechnol. 2011;29:572-3.

32. Olvera C, Goldberg JB, Sánchez R, Soberón-Chávez G. The Pseudomonas aeruginosa algC gene product participates in rhamnolipid biosynthesis. FEMS Microbiol Lett. 1999;179(1):85-90.

33. Rahim R, Burrows LL, Monteiro MA, Perry MB, Lam JS. Involvement of the $\mathrm{rml}$ locus in core oligosaccharide and $\mathrm{O}$ polysaccharide assembly in Pseudomonas aeruginosa. Microbiology. 2000;146(11):2803-14.

34. Reis RS, Pereira AG, Neves BC, Freire DMG. Gene regulation of rhamnolipid production in Pseudomonas aeruginosa - a review. Bioresour Technol. 2011;102:6377-84.

35. Dobler L, Vilela LF, Almeida RV, et al. Rhamnolipids in perspective: gene regulatory pathways, metabolic engineering, production and technological forecasting. New Biotechnol. 2016;33(1):123-35.

36. Long $X$, Shen $C$, He N, et al. Enhanced rhamnolipids production via efficient foam-control using stop valve as a foam breaker. Bioresour Technol. 2017;224:536-43.

\section{Publisher's Note}

Springer Nature remains neutral with regard to jurisdictional claims in published maps and institutional affiliations.

Ready to submit your research? Choose BMC and benefit from:

- fast, convenient online submission

- thorough peer review by experienced researchers in your field

- rapid publication on acceptance

- support for research data, including large and complex data types

- gold Open Access which fosters wider collaboration and increased citations

- maximum visibility for your research: over 100M website views per year

At BMC, research is always in progress.

Learn more biomedcentral.com/submissions 